\title{
Diseño de un sistema perfilador de bajo costo y efectivo para la determinación de rugosidad en vías terrestres asfaltadas
}

\section{Cost-Effective system design for an asphalted-highway roughness profiler}

\author{
GUTIERREZ-VILLALOBOS, Jose M.†*, MORENO-MARTÍNEZ, Jatziri Yunuén, CHAVEZ- \\ CARDENAS, Xhavier y ARROYO-CHÁVEZ, Hiram
}

Universidad de Guanajuato, Campus Celaya-Salvatierra, Av. Javier Barros Sierra 201 Col. Ejido de Santa María del Refugio C.P. 38140 Celaya, Gto. México

ID $1^{\mathrm{er}}$ Autor: José Marcelino, Gutiérrez-Villalobos / ORC ID: 0000-0001-5947-1489, Researcher ID Thomson: S-76662018, CVU CONACYT ID: 173461

ID $1^{\text {er }}$ Coautor: Jatziri Yunuén, Moreno-Martínez / ORC ID: 0000-0002-6798-2067, Researcher ID Thomson: S-8441-2018, CVU CONACYT ID: 237707

ID $2^{\text {do }}$ Coautor: Xavier, Chávez-Cárdenas / ORC ID: 0000-0001-6691-4380, Researcher ID Thomson: F-3210-2018, CVU CONACYT ID: 269911

ID $3^{\text {er }}$ Coautor: Hiram, Arroyo-Chávez / ORC ID: 0000-0002-8343-698X, CVU CONACYT ID: 70975

DOI: $10.35429 / J C E .2019 .10 .3 .10-18$

Recibido 03 de Septiembre, 2019; Aceptado 09 Noviembre, 2019

\section{Resumen}

El constante transitar de camiones con grandes cargas o vehículos demasiado pesado circulando por las vías terrestres del territorio nacional, origina el deterioro prematuro de las carreteras y los caminos pavimentadas con asfalto que conectan al país. Esto además del daño originado por las condiciones climáticas de la zona o región donde se encuentran construidas tales vías de comunicación. Este trabajo esta enfocado al diseño y construcción de un sistema capaz de calcular y estimar el deterioro que ha sufrido una carretera, avenida, calle o camino que estén construidos con superficies asfaltadas, las cuales requieren un mantenimiento periódico y eventualmente se necesita saber su estado físico de manera cuantitativa. Este equipo realiza las mediciones por medio de un sensor que mide le tiempo de vuelo de la luz (laser), mientras un vehículo se desplaza a una velocidad constante sobre la superficie de estudio. A diferencia de la estimación con equipos comerciales que requieren que la medición sea mecánica o física usando la técnica de medio carro enganchado a un vehículo o los perfiladores ópticos que resultan costosos. La contribución de esta propuesta consiste en el diseño de la etapa electrónica para la adquisición de datos y el uso de un sensor óptico económico pero preciso, con el cual se puede calcular con gran precisión el índice de rugosidad, sin la necesidad de un dispositivo mecánico que tenga contacto físico con la superficie. Con la metodología que conlleva, las ecuaciones de diseño, su funcionamiento y validación.

Perfilómetro, IRI, Estimación de rugosidad

\begin{abstract}
The constant transit of trucks with large loads or too heavy vehicles circulating on the roadways of the national territory, causes premature deterioration of the asphalted freeways and roads, which keep country connected. All of this, in addition to the damage caused by the climatic conditions of the area or region where such communication roads are built. This work is focused on the design and construction a system capable of calculating and estimating the deterioration suffered on a road, avenue, street or road that are built with asphalted surfaces, which require periodic maintenance and occasionally their physical condition needs to be known in a quantitative manner. This equipment makes the measurements by means of a sensor that measures the flight time of the light (laser), as a mobile moves at constant speed on the study surface, opposite to the estimation with commercial equipments that require a mechanical or physical measurement by using the technique of half-car attached to a vehicle or the expensive optics profilers. The contribution of this project consists in the design of the electronic stage for data acquisition and the use of an economical but effective optical sensor, which is employed to calculate the roughness index with great precision, without the need for a mechanical device that has physical contact on the surface. With the methodology involving the equations of design, form of operation and validation.
\end{abstract}

Profilometer, IRI, Roughness estimation

Citación: GUTIERREZ-VILLALOBOS, Jose M., MORENO-MARTÍNEZ, Jatziri Yunuén, CHAVEZ-CARDENAS, Xhavier y ARROYO-CHÁVEZ, Hiram. Diseño de un sistema perfilador de bajo costo y efectivo para la determinación de rugosidad en vías terrestres asfaltadas. Revista de Ingeniería Civil. 2019. 3-10: 10-18

\footnotetext{
* Correspondencia del Autor (correo electrónico: jmgutierrez@ugto.mx)

$\dagger$ Investigador contribuyendo como primer autor.
} 


\section{Introducción}

Es muy importante en términos de costos, seguridad y confort, que cualquier usuario de una carretera, camino o calle pavimentada con asfalto, tenga una sensación confortable al conducir a lo largo de la superficie de un camino. En la actualidad las pruebas que se realizan en la mayoría de las carreteras de país, consisten en hacer un recorrido a lo largo de una carretera en la que se desea conocer su estado y es entonces que un conductor se desplaza sobre la vía terrestre a una velocidad constante $y$ va emitiendo un juicio de manera subjetiva y cualitativa y no cuantitativa del estado de la carretera. Sin embargo, oficialmente estas pruebas deben estar basadas en un estándar que determina si el estado del camino se encuentra dentro o fuera de rangos, estas pruebas no aplican para caminos pavimentados con concreto hidráulico, ya que estas vías presentan muy poco o casi nulo deterioro con el tiempo, si embargo si pueden presentar que dada su longitud y construcción se presente el fenómeno resonancia en el desplazamiento de los vehículos a diferentes velocidades, pero eso es tema de otro trabajo.

El estándar utilizado para la verificación del estado de la carreteras, calles o caminos pavimentados con asfalto, es el índice internacional de rugosidad o IRI por sus siglas en Inglés, este índice se basa en recolectar una serie de datos o mediciones sobre la superficie del camino a verificar. Comúnmente el sistema más utilizado para esta tarea es el esquema de $1 / 2$ carro, el cual consiste básicamente en el uso de un neumático sujetado en la parte trasera de un vehículo el cual se desplaza a una velocidad constante, instalado el neumático se encuentra un sensor el cual tiene forma de pistón y tiene una carrera, es decir, se puede entrar o salir una varilla dentro de un cilindro y dicho desplazamiento se convierte en una señal eléctrica la cual se va registrado en un sistema computacional, al final de la prueba dichos datos son graficados y en dicho software se establece el parámetro para determinar a partir de que valor las elevaciones o depresiones se toman como de consideración y finalmente se obtiene un promedio de cuantas señales están fuera de rango por cada kilómetro y en base a cuantos metro por kilómetro se encuentran dañados es que se determina si el camino o carretera esta moco, medio o muy dañado.

\section{Justificación}

Se requiere construir un sistema de bajo costo, preciso y efectivo para medir el nivel de rugosidad que tiene las vías de comunicación terrestres pavimentadas, para emitir un perfil sobre el estado físico del camino y así las autoridades determinen sus mantenimientos de forma periódica.

\section{Problema}

Tener un equipo para medir el IRI no resulta barato y en ocasiones su instalación tampoco es sencilla, desde los sistemas que utilizan mecánicos rodantes colocados al vehículo de inspección como un neumático adicional, conocido como método de $1 / 4$ de carro, en los cuales se instalan los sensores que miden los desplazamientos verticales del neumático para registrar las variaciones del camino, hasta sistemas ópticos que ya no requieren el sistema mecánico rodante pero que son costosos. Por otro lado, el sistema debe funcionar a diferentes velocidades y poder detectar afectaciones como grietas, elevaciones o depresiones.

\section{Hipótesis}

Se puede construir un sistema de bajo costo y efectivo para medir el IR de una vía terrestre asfaltada usando un sensor que mide el tiempo de vuelo de la luz de un láser, instalado en un vehículo.

\section{Objetivos}

\section{Objetivo General}

Desarrollar y construir un sistema de medición de no contacto, que no requiera un sistema mecánico rodante, con base un sensor óptico para medir el tiempo de vuelo de un láser (VL53L0X) y un sistema de adquisición de rápido prototipado con un procesador ATmega2560.

\section{Objetivos específicos}

- Construir el sistema de adquisiciones para las señales de los sensores requeridos como el encoder y el sensor de tiempo de vuelo, utilizando un sistema mínimo de rápido desarrollo. 
Hacer una validación del desempeño del sistema evaluando los perfiles generados a diferentes velocidades.

\section{Marco Teórico}

El IRI se propuso por el Banco Mundial en 1982 (William D. O., 1984), y fue aceptado hasta 1986 (Michael W. and Sayers 1990), en ese índice quedan explicados los pasos para poder determinar el estado de una carretera pavimentada con asfalto, porque también están las pavimentadas con concreto hidráulico, pero este trabajo está enfocado únicamente al pavimento de asfalto. El trabajo que presentaron por Nueraihemaitijiang (2016), presenta una buena propuesta donde se realiza un sistema perfilador en conjunto con el programa de ArcGIS con buenos resultados, utilizando un sistema de tiempo real pero que colecta los datos de la suspensión del vehículo, lo que resulta complicado de instalar.

Por otro lado, la propuesta presentada por Chang etal (2009), emplea un carro autónomo y muestra el uso de un sensor laser, pero el vehículo al ser auto operado, se desplaza a bajas velocidades convirtiéndolo en una alternativa para valoración de instalaciones como edificios o estacionamientos. El resumen de estos sistemas presentado por Kogbara etal (2016), es una buena presentación del estado del arte en el que se encuentran dichos sistemas de estimación y se puede observar por otros trabajos como el de Pawar etal (2018) y Qin etal (2019), de que este tema es aún de interés, ya que en ellos se estudia como la respuesta de oscilación del vehículo está directamente relacionada con la superficie del camino y como algunas modificaciones en la sucesión pueden ayudar a disminuir estas vibraciones.

Otra propuesta similar a la que se presenta en el presente trabajo, es la de Wang etal (2011), donde presentan un sistema para medir la textura el pavimento, pero el equipo utilizado es de un alto costo, su diseño lo hacer adecuado para estudio de tramos con superficies cortas, como una calle o avenida. Oldrich Kropa and Peter Muckab 2005, nos previenen de no considerar el IRI como un indicador de desnivel de la superficie de un camino, esto es importante porque se debe hacer una correcta selección de parámetro a observar en las carreteras o autopistas, ya que las elevaciones juegan un papel importante en el confort y seguridad de los usuarios.

\section{Sensor de tiempo de vuelo}

Este dispositivo VL53L0X, llamado medidor de tiempo de vuelo o ToF por sus siglas en inglés el cual se observa en la figura 1 , resulta vital, para la construcción de esta propuesta y es la pregunta clave para este trabajo, si es que podrá medir la variaciones de elevación de la superficie, permite medir o calcular la distancia de un objeto dentro de un rango absoluto de hasta 2 metros (en condiciones ideales), dicho sensor hace uso del tiempo que le lleva a un haz de luz (imperceptible por la vista humana) reflectarse sobre una superficie y ser detectado, con una resolución de hasta 1 milímetro, gracias a un su sistema de compensación óptico embebido y cuenta con una salida de comunicación con protocolo I2C.

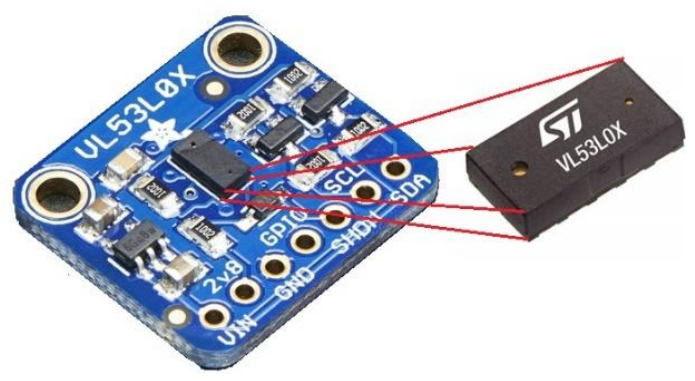

Figura 1 Sensor de tiempo de vuelo VL53L0X

El ángulo de apertura que tienen tanto el emisor como el receptor es de $\alpha=35^{\circ}$ y $\beta=25^{\circ}$ respectivamente, que debe ser tomado en cuenta para su colocación del módulo dentro de los que es su caja de colocación y transporte para que no interfiera con su funcionamiento., además para calcular el área de cobertura del sensor como se verá más adelante. Cuando inicia el equipo, el sistema esta esperado que el usuario realice una medición inicial (la que hemos denominado altura absoluta (botón de Tara en la aplicación del dispositivo móvil) lo que se puede apreciar en la figura $2 \mathrm{a}$, la cual se resta a las mediciones siguientes llamadas alturas relativas y con esta diferencia se obtiene la altura actual, de esa forma se detectan las deformaciones 0 afectaciones de la superficie, cuando la altura o distancia actual medida por el sensor (después de la distancia absoluta) es negativa esto quiere decir que la distancia actual es inferior que la distancia inicial y por lo tanto se ha detectado una cresta (figura 2b), en cabio si en la medición la distancia es superior a la inicial y la diferencia es positiva, esto quiere decir que se ha encontrado un valle(figura 2c). Esto queda explicado en la ecuación 1. 


$$
H_{\text {actual }}=H_{\text {relativa }}-H_{\text {absoluta }}
$$

\section{Donde:}

$\mathrm{H}_{\text {actual }}=$ la altura real de la falla en $\mathrm{mm}$

$\mathrm{H}_{\text {trelativa }}=$ la altura de la lectura actual en $\mathrm{mm}$

$\mathrm{H}_{\mathrm{absoluta}}=$ la altura inicial hacia la superficie en $\mathrm{mm}$

En condiciones de exterior, con luz y el color de la superficie del camino puede disminuir su rango precisión hasta en $12 \%$, por lo que existe la necesidad de caracteriza el sensor $\mathrm{y}$ ver sus mediciones bajo diferentes condiciones de operación, como cantidad de luz solar (hora del día), el color del pavimento y la altura a la que de debe colocar el sensor.

Se utilizan 2 sensores para cubrir un área de observación mayor al mismo tiempo, debido a que cada uno se encuentra a una altura de 25 $\mathrm{cm}$ sobre la superficie, el área que cada uno cubren es de aproximadamente $22 \mathrm{~cm}$ cada uno, los sensores están a una separación $60 \mathrm{~cm}$ entre ellos, el área de cobertura de cada sensor está dada por la ecuación (2).

$$
A_{\text {cobertura }}=D_{\text {sensor }} *\left(\frac{\operatorname{sen}\left(\frac{\beta}{2}\right)}{\cos \left(\frac{\beta}{2}\right)}\right)
$$

Donde:

ACOBERTURA $=$ área de cobertura del sensor en $\mathrm{cm} 2$

$D_{\text {sensor }}=$ altura a la que se coloca el sensor en $\mathrm{cm}$ $\beta=$ ángulo en grados de apertura del colector del sensor

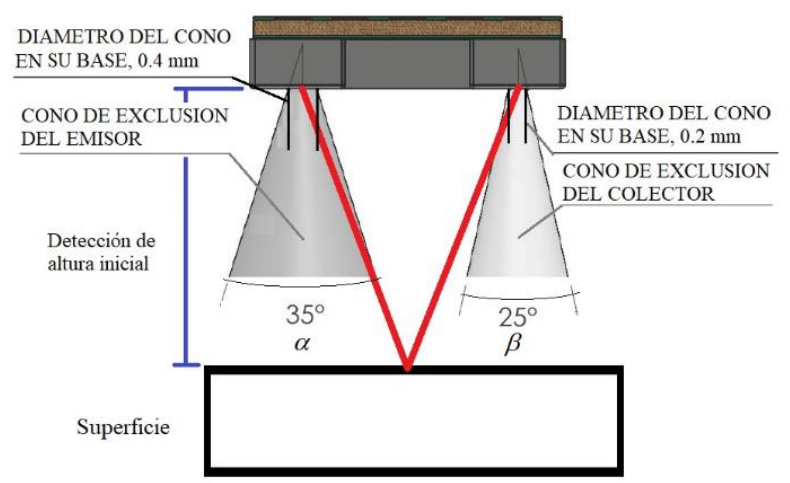

a)

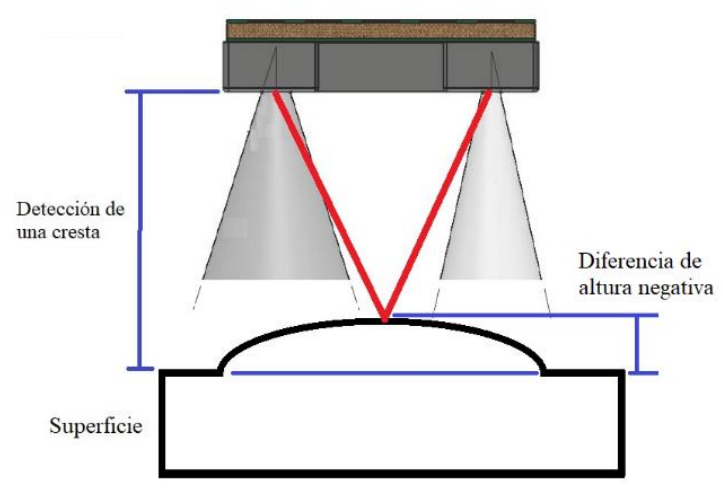

b)

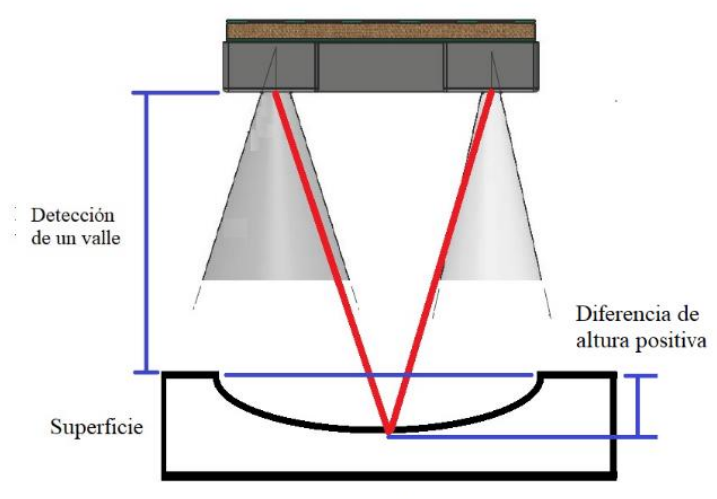

c)

Figura 2 Ilustración de como se obtienen las afectaciones sobre la superficie del camino

\section{Sistema de adquisición}

El sistema completo está conformado por un sistema mínimo, sin necesidad de una computadora. Dicho sistema es una tarjeta de rápida implementación basada en un microcontrolador ATmega2560, el cual tiene un cristal de $16 \mathrm{MHz}$, una memoria Flash de 256 $\mathrm{KB}$, una memoria SRAM de $8 \mathrm{~KB}$ y una memoria EEPROM de $4 \mathrm{~KB}$. Adicionalmente tiene 3 juegos de comunicación serial de los cuales usamos uno para la conexión del módulo Bluetooth, tiene puerto de comunicación I2C que es por donde se comunican los 2 sensores y el reloj de tiempo real, un conjunto de puertos para la comunicación SPI por la cual se comunica el modulo de la tarjeta lectora Micro SD.

GUTIERREZ-VILLALOBOS, Jose M., MORENO-MARTÍNEZ, Jatziri Yunuén, CHAVEZ-CARDENAS, Xhavier y ARROYO-CHÁVEZ, Hiram. Diseño de un sistema perfilador de bajo costo y efectivo para la determinación de rugosidad en vías terrestres asfaltadas. Revista de Ingeniería Civil. 2019 
Las interconexiones electrónicas se pueden ver el diagrama de la figura $3 \mathrm{a}$, mientras que la figura $3 \mathrm{~b}$ se ve físicamente como están interconectados los elementos del circuito y en la figura $3 \mathrm{c}$, se observan los conectores para los sensores VL53L0X, el encoder y la alimentación.

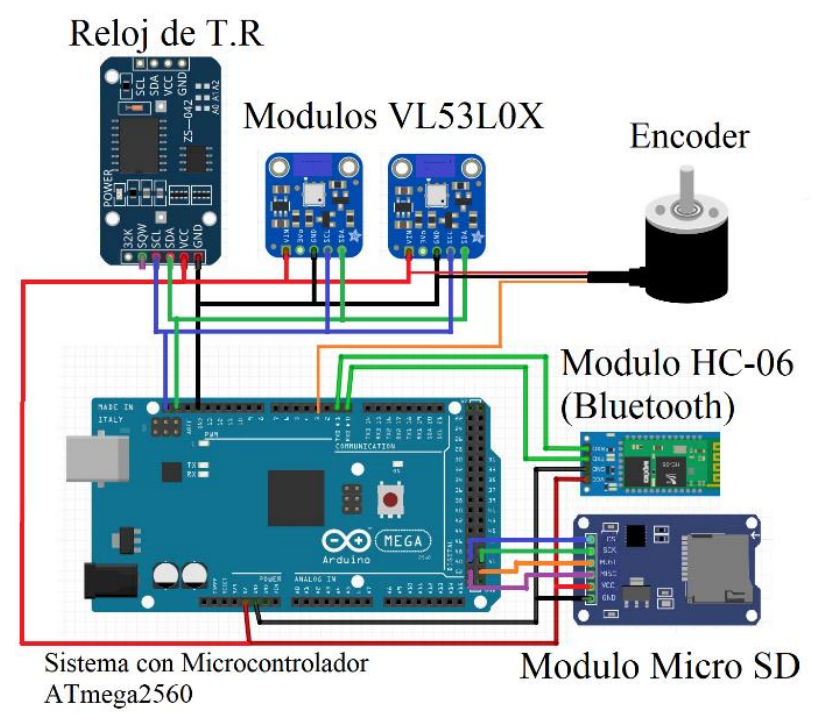

a)

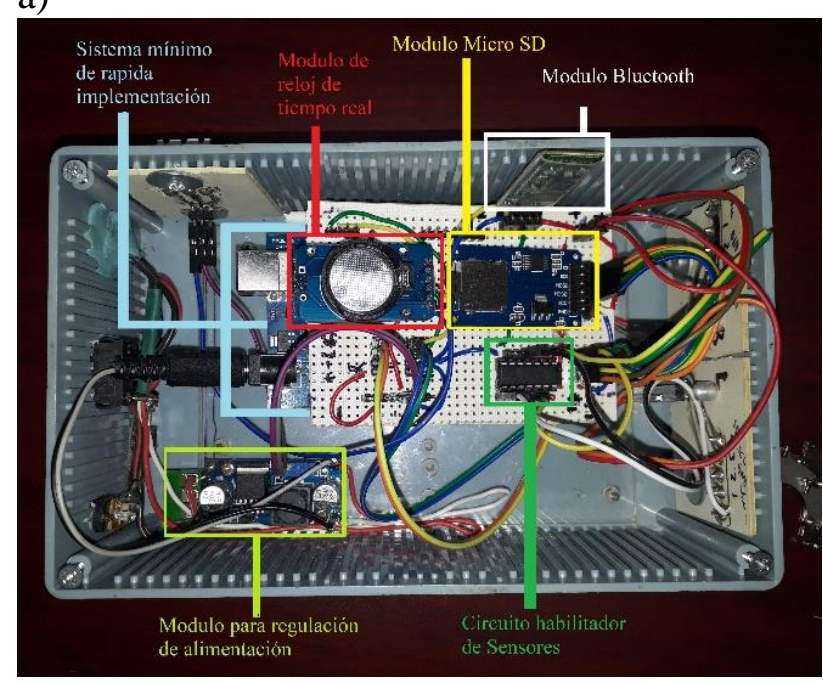

b)

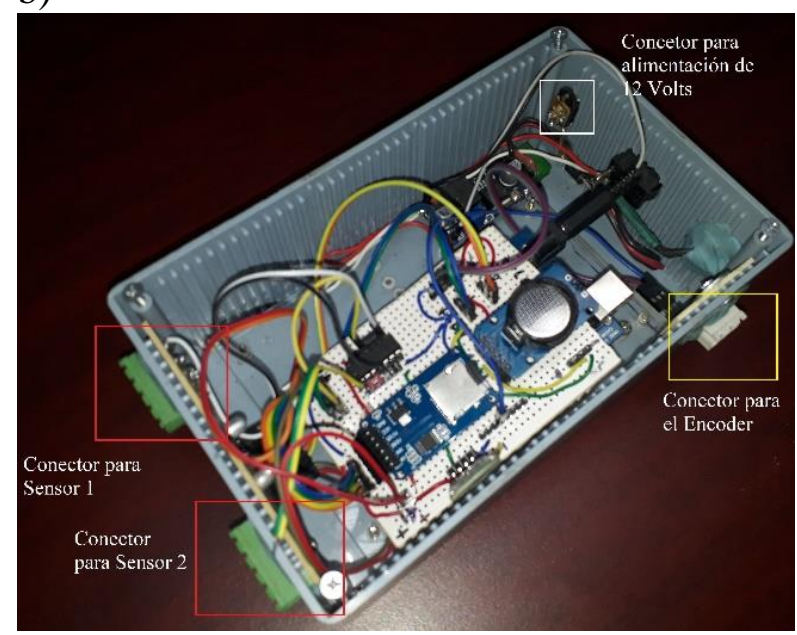

c)

Figura 3 Sistema perfilador, a) diagrama de interconexión electrónica, b) colocación de los diferentes elementos y c) conectores para los dispositivos externos, respectivamente

\section{Operación del sistema}

La interface para controlar el sistema es una aplicación desarrollada para este proyecto MIT inventor, por medio de comunicación bluetooth con un dispositivo móvil, una vez iniciada la comunicación y con el vehículo estático, se introduce el radio de la llanta de la camioneta, esto determinara el desplazamiento de la llanta por cada revolución, posterior se introduce el periodo de muestreo, esto es a que distancia sebe realizar la medición de la altura de los sensores, la cual puede ser a $1 / 4$ de metro, $1 / 2$ metro o 1 metro.

Aun sin comenzar el avance, se presiona el botón tara, que determinara la altura existente sobre la superficie lo más lineal posible, esta se convierte en altura absoluta como explica en secciones anteriormente. La figura 4, muestra la interface de control, para el perfilador, por ultimo los botones de off y on son para que se inicie y se detenga la adquisición de alturas. En el recuadro debajo de los botones se va mostrando el avance del vehículo que se tiene en el momento.

IRI Measurer
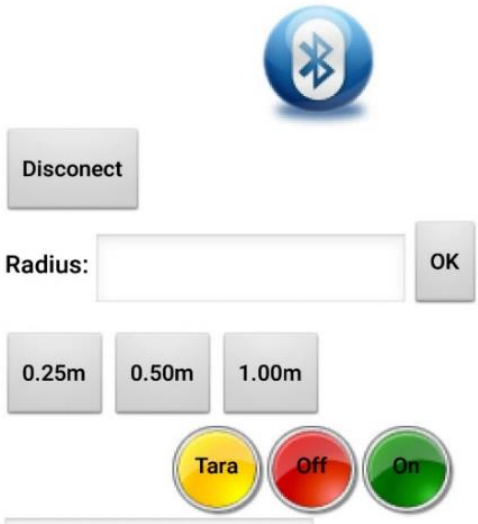

Figura 4 Interface de control del perfilador.

Las mediciones de altura registradas con el perfilador, quedan selladas con la fecha y la hora del reloj de tiempo real y son almacenadas en la memoria SD, para su posterior procesamiento. El tiempo real es necesario, ya que también se graba en video el recorrido realizado, para su revisión y análisis. En caso de encontrar anomalías de una profundidad o altura superior a la que se determine como permitida por el usuario, con el video y por el tiempo, se pude buscar y ubicar las afectaciones que se presenten en las gráficas. 


\section{Determinación del IRI}

Para la determinación del índice de rugosidad se realiza por medio de la suma las alturas (valor absoluto) registradas por el sensor de tiempo de vuelo y almacenadas en el sistema, luego, este valor acumulado se divide entre a longitud que se usa como referencia, por ejemplo, $\mathrm{m} / \mathrm{km} \mathrm{o}$ $\mathrm{in} / \mathrm{mi}$ y el resultado de ese cociente es el que determina al índice de rugosidad de dicha longitud o tramo de acuerdo a los valores establecidos por el IRI. Como se observa en la ecuación (3), presentada por Sayers and Michael W., (1995). Dicho índice puede observarse en la figura 5 .

$$
I R I=\frac{\sum_{i=1}^{n}\left|Y_{i}\right| *(0.01)}{D} \quad(\mathrm{~m} / \mathrm{km})
$$

Donde

$Y_{i}=$ Valor de cada medición positiva o negative registrada por el sistema en $\mathrm{mm}$.

$D=$ Longitud de referencia en $\mathrm{km}$.

Esta escala será útil para el análisis posterior de los caminos recorrido y valorados por este sistema.

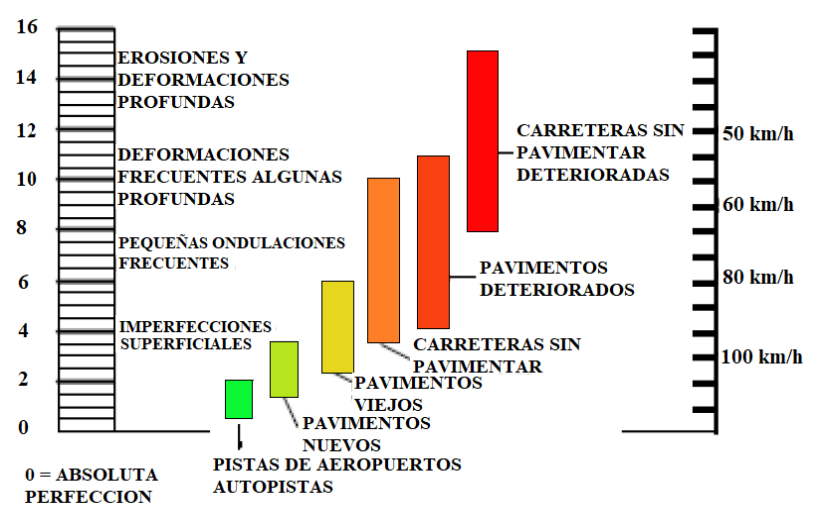

Figura 5 Escala del IRI y los estados generales de la superficie del pavimento

Estos valores se pueden observar en la tabla 1, que contiene los valores o escalas del IRI.

\begin{tabular}{|l|r|}
\hline Estado del camino & $\begin{array}{r}\text { Pavimento con asfalto } \\
\text { IRI (m/km) }\end{array}$ \\
\hline Muy bueno & $<3.2$ \\
\hline Bueno & $3.2-3.9$ \\
\hline Regular & $4.0-4.6$ \\
\hline Malo & $>4.6$ \\
\hline
\end{tabular}

Tabla 1 Valores de IRI para una avenida asfaltada

\section{Configuración del sistema}

En la imagen 6, se muestra cómo esta monta todo el equipo de medición sobre el vehículo, el encoder va en la llanta trasera derecha viéndolo desde atrás, los sensores van en una barra que permite colocaros a la distancia necesaria para que no interfieran entre ellos, pero tampoco permitir que la separación sea muy grande y se escapen las deformaciones de la superficie, aquí se dejaron a $60 \mathrm{~cm}$ de separación.

También se puede ajustar su altura con respecto la superficie del suelo, que en este caso fue de $25 \mathrm{~cm}$. La cámara va montada en un brazo lo que la deja a una altura de $1.7 \mathrm{~m}$, altura suficiente para que el ángulo de apertura de la cámara pueda grabar correctamente toda la superficie recorrida, la cámara utilizada es una cámara SONY modelo DSC-RXOG, que es una cámara de alta velocidad.

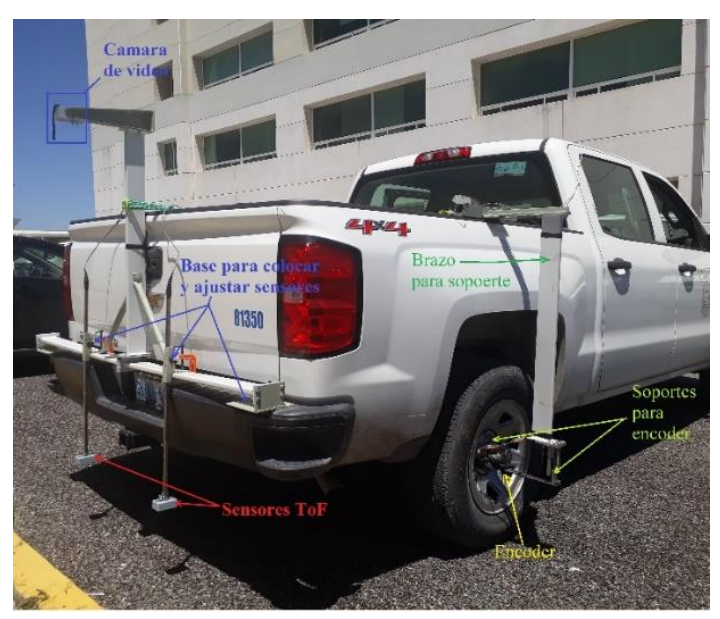

Figura 6 Configuración del sistema montado en el vehículo de prueba

\section{Resultados}

Con el sistema instalado en el vehículo, se hicieron varios recorridos por carreteras pavimentadas. Como se mencionó anteriormente, el uso de los 2 sensores obedece a cubrir más área con las mediciones, ellos se encuentran a la misma altura, por lo que se esperaba que las lecturas entre ambos sean similares, más no idénticas, porque las afectaciones son diferentes durante el trayecto. Para validar la propuesta se presentan imágenes de diversos trayectos recorridos, dichos trayectos se efectuaron en carreteras diferentes. 
Primeramente, se hizo el recorrido sobre una carretera de $10 \mathrm{~km}$ relativamente nueva o en muy buen estado, con algunas grietas, pero la superficie generalmente pareja, con una velocidad promedio de $80 \mathrm{~km} / \mathrm{h}$ y un índice de muestreo de $0.5 \mathrm{~m}$, dado que el IRI se calcula en tramos de un kilómetro, se tendrían 10 tramos para este ensayo, a lo que se presenta uno de estos tramos con sus respectivos 2000 valores capturados. Con estos datos y los limites inferior y superior respectivamente, se lleva a cabo el calculo del IRI por cada sensor y su respectiva grafica como se muestra en la figura 7 , donde observar el sensor derecho en la figura $7 \mathrm{a}$, el sensor izquierdo en la figura $7 \mathrm{~b}$ y finalmente con ayuda de la cámara es que se puede observar una foto del estado general de un tramo sobre la carretera recorrida y se pueden comparar los resultados de ambos sensores, al igual que la similitud entre el IRI de los dos.
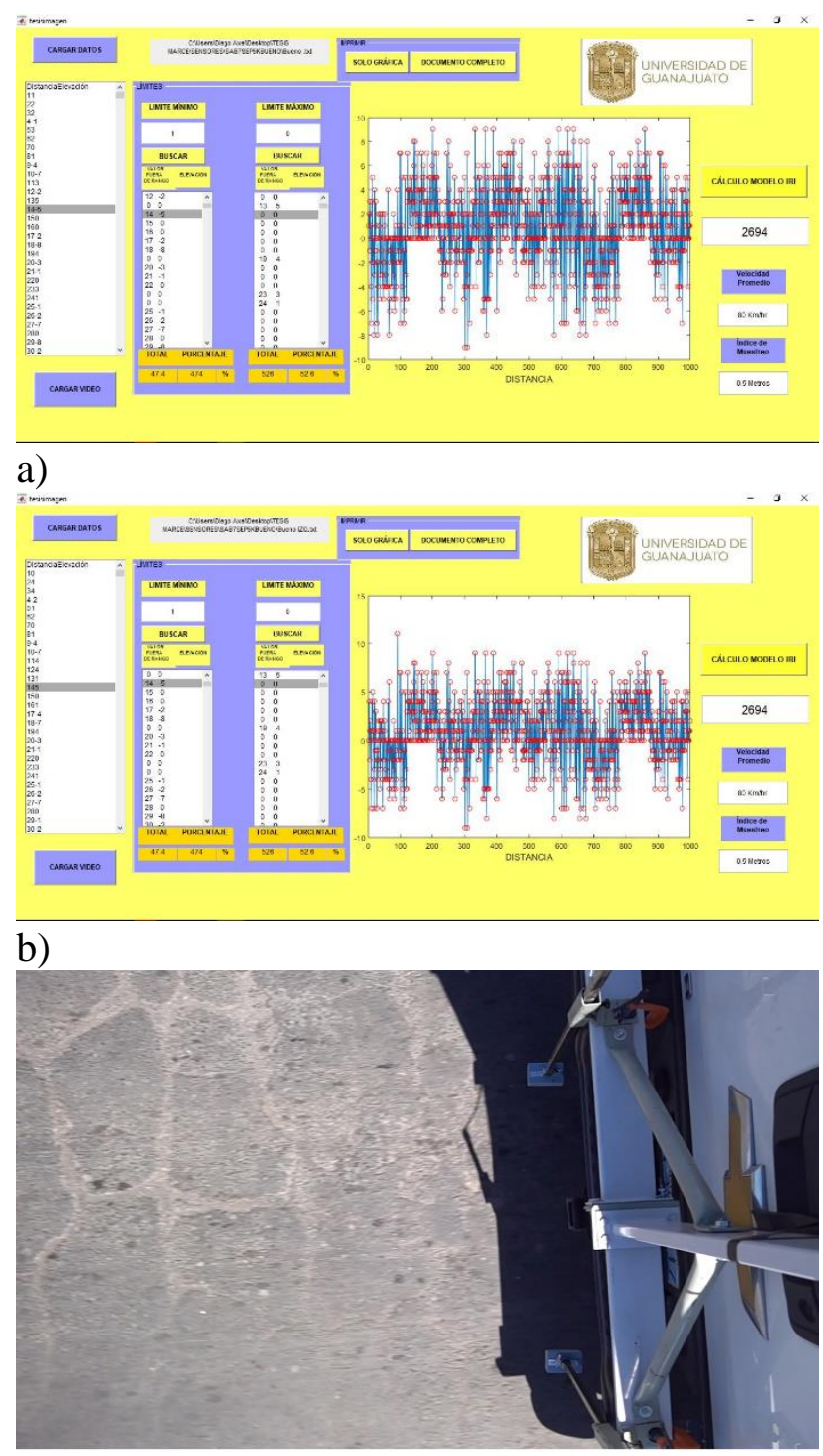

c)

Figura 7 Tramo de un camino con regular estado, con una velocidad promedio de $80 \mathrm{~km} / \mathrm{k}$, un índice de muestro de $0.5 \mathrm{~m}$, a) sensor derecho, b) sensor izquierdo y c) foto de la superficie recorrida
En la figura 8, se muestra un tramo de otra carretera que es visible su deterioro y las afectaciones sobre la superficie, en este caso también este recorrido se realizó a $80 \mathrm{~km} / \mathrm{h}$ con el mismo índice de muestreo de $0.5 \mathrm{~m}$. También se presentan ambos sensores en un tramo de 1 $\mathrm{km}$ y sus 2000 muestras y se puede observar la similitud entre ambas gráficas y ambos IRI, además de una foto del estado de la superficie.

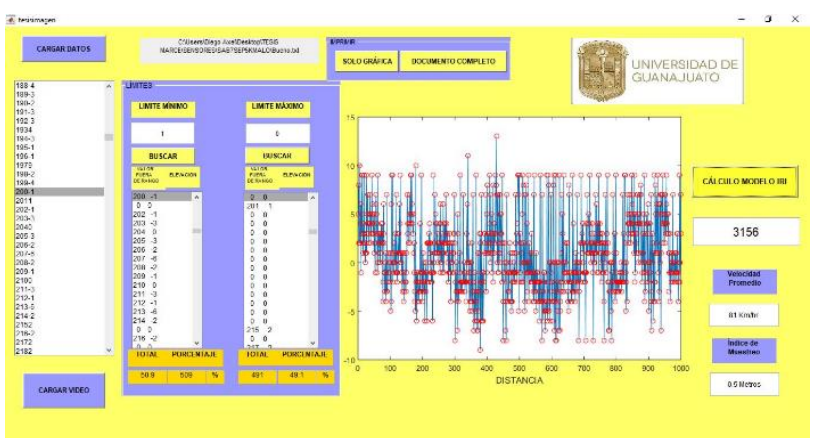

a)

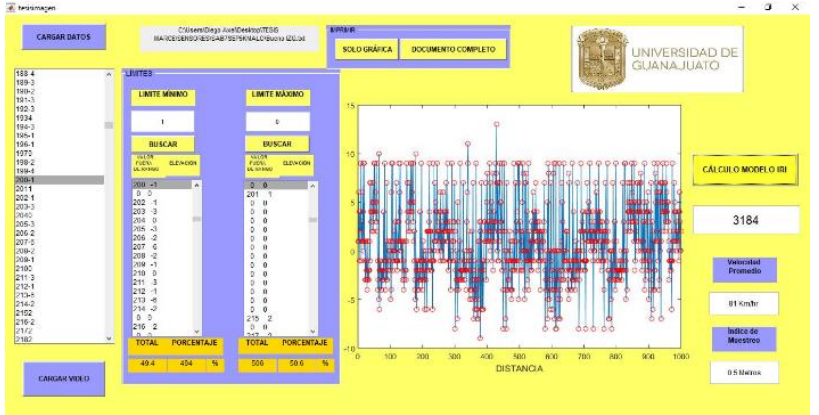

b)

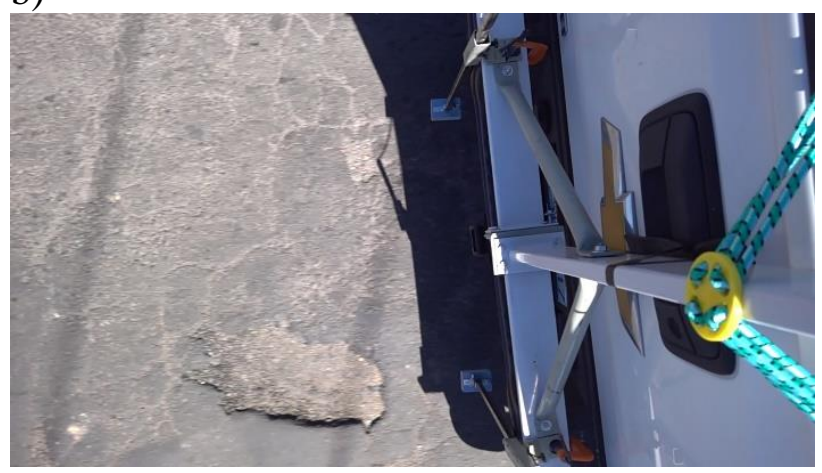

c)

Figura 8 Tramo de un camino con notable deterioro a simple vista, con una velocidad promedio de $80 \mathrm{~km} / \mathrm{k}$, un índice de muestro de $0.5 \mathrm{~m}$, a) sensor derecho, b) sensor izquierdo y c) foto de la superficie recorrida

Finalmente, para comprobar el funcionamiento y desempeño del sistema, se hizo un recorrido por una tercera carretera pavimentad con una superficie también de regulares condiciones, en este caso se presenta el mismo tramo del recorrido en el mismo kilómetro, un periodo de muestreo también de $0.5 \mathrm{~m}$, pero a diferentes velocidades.

GUTIERREZ-VILLALOBOS, Jose M., MORENO-MARTÍNEZ, Jatziri Yunuén, CHAVEZ-CARDENAS, Xhavier y ARROYO-CHÁVEZ, Hiram. Diseño de un sistema perfilador de bajo costo y efectivo para la determinación de rugosidad en vías terrestres asfaltadas. Revista de Ingeniería Civil. 2019 
En la figura 9 a) y b) se observa las gráficas del sensor derecho y del sensor izquierdo respectivamente de dicho kilometro a una velocidad promedio de $20 \mathrm{~km} / \mathrm{h}$.

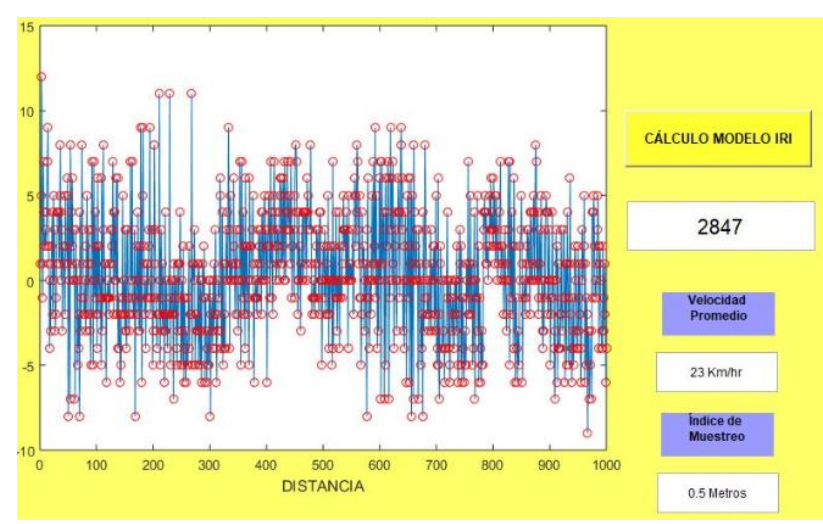

a)

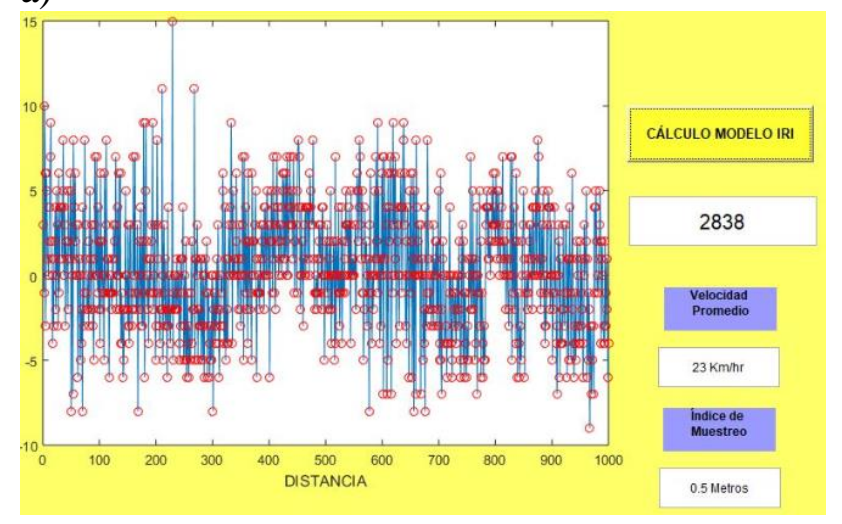

b)

Figura 9 Tramo de un camino con algo de deterioro a simple vista, con una velocidad promedio de $20 \mathrm{~km} / \mathrm{k}$, un índice de muestro de $0.5 \mathrm{~m}$, a) sensor derecho y b) sensor izquierdo

En la figura 10 a) y b) se observa las gráficas del sensor derecho y del sensor izquierdo también del mismo kilometro a una velocidad promedio de $50 \mathrm{~km} / \mathrm{h}$. Por último, en la figura 10 a) y b) se observa las gráficas del sensor derecho y del sensor izquierdo nuevamente del mismo kilometro a una velocidad promedio de $80 \mathrm{~km} / \mathrm{h}$.

Los resultados mostraron una buena respuesta de las mediciones que se efectuaron sobre una misma carpeta asfaltada en el mismo tramo, de un trayecto de $10 \mathrm{~km}$, pero se puede observar como a mayor velocidad las mediciones cambian un poco, pero esto obedece principalmente al efecto de la resonancia del periodo de camino con la velocidad del vehículo. Pero se puede determinar que a velocidades medias el cálculo del IRI es muy semejante y que a altas velocidades también es cercano, pero si se ve afectado.

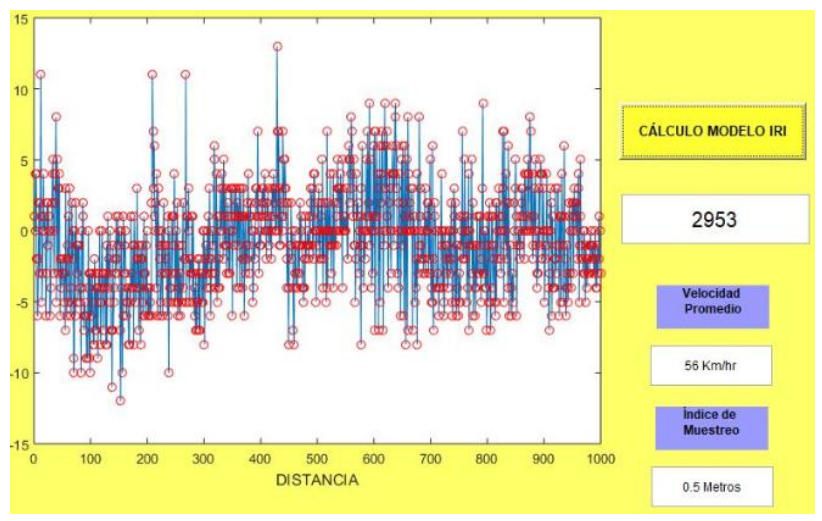

a)

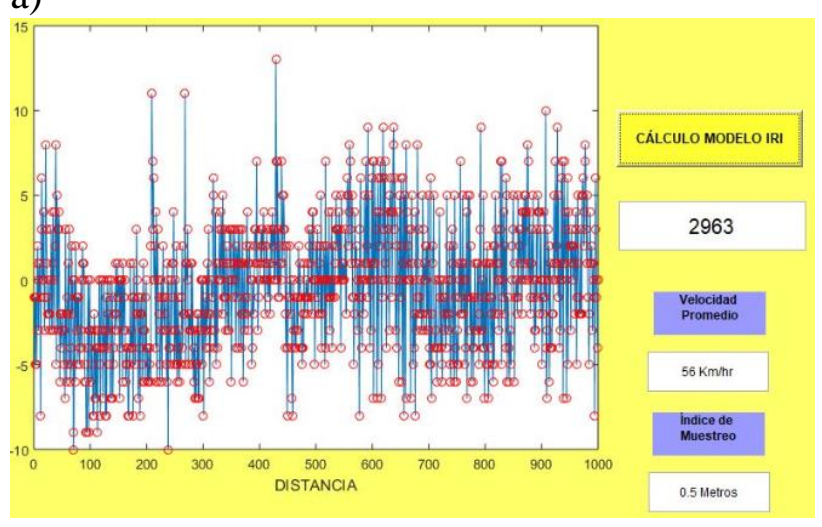

b)

Figura 10 Tramo de un camino con algo de deterioro a simple vista, con una velocidad promedio de $50 \mathrm{~km} / \mathrm{k}$, un índice de muestro de $0.5 \mathrm{~m}$, a) sensor derecho y b) sensor izquierdo

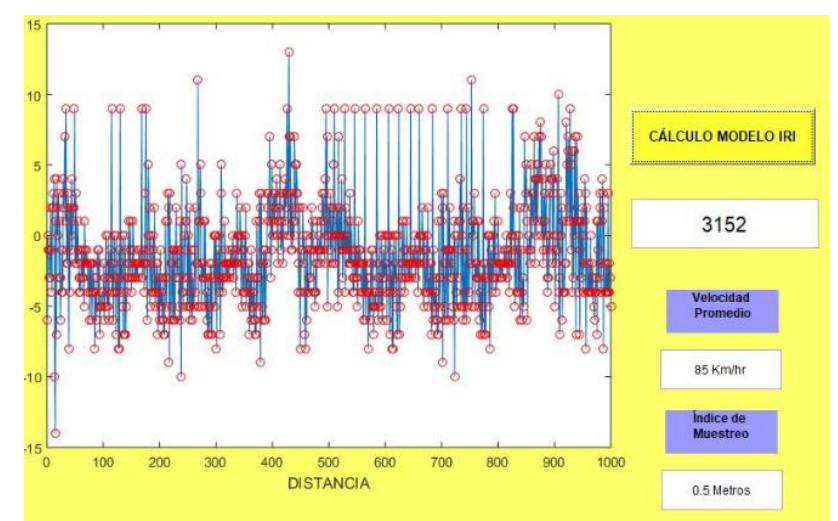

a)

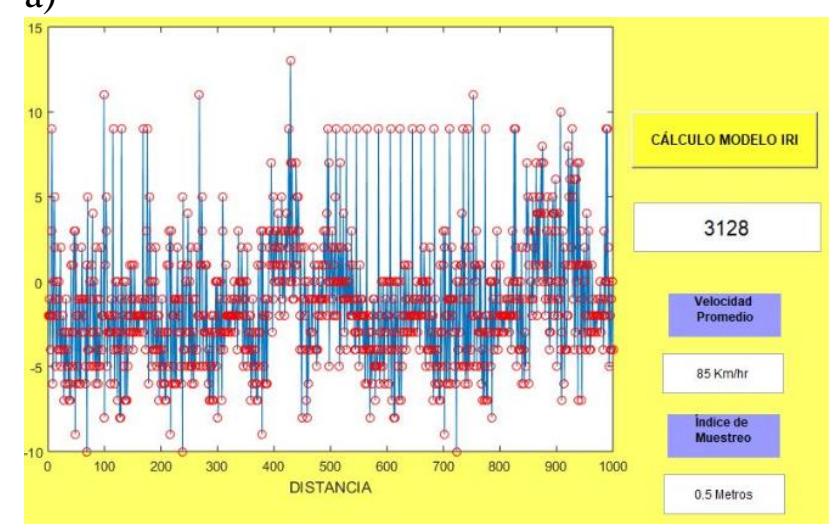

b)

Figura 11 Tramo de un camino con algo de deterioro a simple vista, con una velocidad promedio de $80 \mathrm{~km} / \mathrm{k}$, un índice de muestro de $0.5 \mathrm{~m}$, a) sensor derecho y b) sensor izquierdo 


\section{Conclusiones}

Se puede concluir de los resultados aquí presentados, que el sensor para medir el tiempo de vuelo propuesto en este trabajo, puede realizar la tarea de estar efectuando mediciones verticales aun cuando este se encuentra bajo un desplazamiento horizontal. Un parámetro que puede afectar de manera considerable los resultados será la velocidad de desplazamiento, pero principalmente por el fenómeno de resonancia, porque se puede observar que aun a $80 \mathrm{~km} / \mathrm{h}$ el sistema puede efectuar mediciones cercanas a las realizadas con bajas y medias velocidades. Los valores de IRI obtenidos a medias y bajas velocidades son muy cercanas.

Es importante comentar que el sistema mínimo basado en el microcontrolador ATmega2560 también fue suficiente para la adquisición de las señales del enconder, los 2 sensores, accesar al reloj de tiempo real y guardar los datos en una memoria microSD sin perder los datos a un índice de muestro mínimo de $0.5 \mathrm{~m}$ y de $1 \mathrm{~m}$.

\section{Agradecimientos}

Los autores agradecen a la Universidad de Guanajuato por el apoyo y las facilidades brindadas. El primer autor agradece a la convocatoria CIIC2019.

\section{Referencias}

Jia-Ruey Chang, Yung-Shuen Su, Tsun-Cheng Huang, Shih-Chung Kang and Shang-Hsien Hsieh, "Measurement of the International Roughness Index (IRI) Using an Autonomous Robot (P3-AT)", 26th International Symposium on Automation and Robotics in Construction (ISARC 2009).

Michael W. and Sayers, "Profiles of Roughness", Measurement of pavement surface condition 1990, Transportation Research Record 1260.

Nueraihemaitijiang Abulizi, Akira Kawamura, Kazuya Tomiyama and Shun Fujita, "Measuring and evaluating of road roughness conditions with a compact road profiler and ArcGIS", Journal of Traffic and Transportation Engineering (English Edition), 2016, vol. 3, 5, pp. 398-411.
Oldrich Kropa and Peter Muckab, "Be careful when using the International Roughness Index as an indicator of road unevenness", Journal of Sound and Vibration, 2005, vol. 287, pp. 9891003.

Prashant R Pawar, Dr Arun Tom Mathew and M R Saraf, "IRI (International Roughness Index): An Indicator Of Vehicle Response", Materials Today: Proceedings 2018, Vol. 5, pp. 1173811750.

Reginald B. Kogbara, Eyad A. Masad, Emad Kassem, A. (Tom) Scarpas and Kumar Anupam, "A state-of-the-art review of parameters influencing measurement and modeling of skid resistance of asphalt pavements", Construction and Building Materials, 2016, vol. 114, pp. 602617.

Sayers and Michael W, "On the Calculation of International Roughness Index from Longitudinal Road Profile", Pavement-vehicle interaction and traffic monitoring 1995, Transportation Research Record 1501.

Weifeng Wang, Xinping Yan a,c, Helai Huang, Xiumin Chu and Mohamed Abdel-Aty, "Design and verification of a laser based device for pavement macrotexture measurement", Measurement of pavement surface condition 2011, Transportation Research Part C, 2011, Vol.19, pp. 682-694.

William D. O. and Paterson, "International Roughness Index: Relationship to Other Measures of Roughness and Riding Quality", 65th Annual Meeting of the Transportation Research Board 1981, Transportation Research Record 1084.

Yechen Qin, Zhenfeng Wang, Changle Xiang, Ehsan Hashemi, Amir Khajepour, Yanjun Huang, "Speed independent road classification strategy based on vehicle response: Theory and experimental validation", Mechanical Systems and Signal Processing, 2019, Vol 117, pp. 653666 